\title{
Reduced inhibition in depression impairs stimulus processing in human cortical microcircuits
}

\author{
Heng Kang Yao ${ }^{1,2, *}$, Alexandre Guet-McCreight ${ }^{1, *}$, Frank Mazza $^{1,2}$, Homeira Moradi Chameh $^{3}$, \\ Thomas D. Prevot ${ }^{4,5}$, John Griffiths ${ }^{1,4,6}$, Shreejoy J. Tripathy ${ }^{1,4,6}$, Taufik A. Valiante ${ }^{3,6,7,8,9}$, \\ Etienne Sibille ${ }^{4,5,10}$, Etay Hay ${ }^{1,2,4}$ \\ ${ }^{1}$ Krembil Centre for Neuroinformatics, Centre for Addiction and Mental Health ${ }^{2}$ Department of Physiology, University of Toronto ${ }^{3}$ Krembil \\ Research Institute, University Health Network ${ }^{4}$ Department of Psychiatry, University of Toronto ${ }^{5}$ Campbell Family Mental Health Research \\ Institute, Centre for Addiction and Mental Health ${ }^{6}$ Institute of Medical Sciences, University of Toronto ${ }^{7}$ Department of Electrical and Computer \\ Engineering, University of Toronto ${ }^{8}$ Institute of Biomaterials and Biomedical Engineering, University of Toronto ${ }^{9}$ Department of Surgery, \\ University of Toronto ${ }^{10}$ Department of Pharmacology \& Toxicology, University of Toronto "These authors contributed equally
}

\begin{abstract}
Cortical processing depends on finely-tuned excitatory and inhibitory connections in neuronal microcircuits. Reduced inhibition by somatostatin-expressing interneurons is a key component of altered inhibition associated with treatment-resistant major depressive disorder (depression), which is implicated in cognitive deficits and rumination, but the link remains to be better established mechanistically in humans. Here, we tested the impact of reduced somatostatin interneuron inhibition on cortical processing in human neuronal microcircuits using a data-driven computational approach. We integrated human cellular, circuit and gene-expression data to generate detailed models of human cortical microcircuits in health and depression. We simulated microcircuit baseline and response activity and found reduced signal-to-noise ratio and increased false/failed detection of stimuli due to a higher baseline activity in depression. Our results thus applied novel models of human cortical microcircuits to demonstrate mechanistically how reduced inhibition impairs cortical processing in depression, providing quantitative links between altered inhibition and cognitive deficits.
\end{abstract}

Corresponding author: Dr. Etay Hay

Krembil Centre for Neuroinformatics, Centre for Addiction and Mental Health 250 College St, Toronto, Ontario, M5T 1R8

Email: etay.hay@ camh.ca 


\section{Introduction}

Cortical processing relies on specific interactions between different types of neurons with distinct electrical properties and synaptic connectivity ${ }^{1,2}$. Accordingly, there is increasing evidence that cortical dysfunction involves changes in the cellular and microcircuit properties. Altered cortical inhibition is implicated in a variety of brain disorders such as autism, schizophrenia and major depressive disorder (depression) ${ }^{3-8}$. Reduced dendritic inhibition from SST interneurons is a key component of the altered inhibition that is associated with treatment-resistant depression and several other disorders ${ }^{3,4,7-11}$. Recent findings showed a significantly lower SST expression by SST interneurons in post-mortem cortical tissue from depression patients, which indicates a reduction in SST inhibition in depression ${ }^{11}$. Accordingly, silencing SST inhibition in rodents produced anxiety and depression symptoms, and new pharmacology boosting SST inhibition led to procognitive and antidepressant effects ${ }^{10,12}$. However, the link between reduced SST inhibition and cortical deficits remains to be better established mechanistically, particularly in humans.

In the cortex, SST interneurons primarily target the apical dendrites of pyramidal (Pyr) neurons and provide synaptic and extra-synaptic (tonic) inhibition through activation of alpha-5gamma-aminobutyric-acid-A $\left(\alpha 5-\mathrm{GABA}_{\mathrm{A}}\right)$ receptors ${ }^{2,13}$. SST interneurons mediate lateral inhibition of Pyr neurons through inhibitory disynaptic loops, where facilitating excitation from a Pyr neuron is sufficient for triggering spikes in an SST interneuron and consequently inhibit neighbouring Pyr neurons ${ }^{14,15}$. At the microcircuit level, SST interneurons are involved in maintaining a low baseline activity of Pyr neurons, but are largely silent during the early response of the cortical microcircuit to stimuli ${ }^{1}$. The role of SST interneurons in baseline activity was further supported by a two-fold increase in the baseline firing rate of Pyr neurons when SST interneurons were silenced ${ }^{1}$.

In line with these findings, a leading hypothesis suggests that a reduced SST inhibition in depression would increase the baseline cortical activity (noise) while minimally impacting stimulus response (signal) and thus decrease the signal to noise ratio (SNR) of cortical processing ${ }^{4}$. This is further supported by studies in animal models of depression, where positive allosteric modulation of $\alpha 5-\mathrm{GABA}_{\mathrm{A}}$ receptors (which are targeted by SST interneurons) led to pro-cognitive effects and recovery from depression symptoms in rodents ${ }^{10,12}$. However, it remains unclear whether the level of reduced SST interneuron inhibition estimated from gene expression data in human depression would have a significant effect on baseline activity and thus on cortical 
processing. In addition, the effect of reduced SST interneuron inhibition on Pyr neuron baseline and response activity is difficult to predict due to the inter-connectivity between the different neuron types in the microcircuit. Moreover, the implications of reduced inhibition on processing SNR and signal detection in depression remain to be determined, to better link the cellular and circuit effects to specific cognitive deficits in depression such as patients being less able to identify relevant signals from noise ${ }^{16}$, or unable to suppress loops of internal thoughts in rumination ${ }^{3}$.

The link between reduced SST interneuron inhibition and depression is supported by preclinical animal models ${ }^{7,10,12}$, but there is a need for mechanistic studies to assess whether this translates to humans. There are cellular and circuit similarities between rodents and humans ${ }^{17}$, e.g. in the intrinsic firing properties and connectivity patterns of cell types, but also important differences. Human inhibitory synapses from SST and parvalbumin (PV) interneurons onto Pyr neurons are stronger, with lower probability of synaptic failures and larger PSP amplitudes compared to rodents ${ }^{14,18,19}$. The connection probability between Pyr neurons in human layer 2/3 (L2/3) is also significantly higher than in rodents ${ }^{20}$. Furthermore, human Pyr neuron and interneuron morphologies are larger than in rodents, with longer and more complex dendrites allowing for more complex signal integration ${ }^{21,22}$, and a more compartmentalized apical dendritic tree ${ }^{23}$. As such, it is imperative to study the mechanisms of dysfunctional cortical processing in depression in the context of human microcircuits.

Computational models are well suited to bridge this gap, due to limitations in monitoring human neuron types and microcircuits in vivo and due to the increasing data availability of neuronal and synaptic recordings in healthy human cortical slices resected during surgery, especially from cortical L2/3 ${ }^{14,20,24}$. These novel data can be integrated into detailed models of human cortical microcircuits to study human cortical processing mechanistically in health and disease. Available models of human L2/3 neurons reproduced some of the firing properties ${ }^{25}$, but only partly reproduced the frequency-input relationship. These models were not constrained with sag-current properties, which depend on dendritic h-current, and thus were limited in capturing dendritic input integration properties of the neurons. In addition, there are currently no models for human synaptic connections and cortical microcircuits. Therefore, there remains a need to integrate the available human electrophysiological data into detailed cortical microcircuit models, and their baseline and response activity. 
In this study, we tested whether the reduced SST inhibition estimated from gene expression in depression results in significant changes in the SNR of human cortical processing and in the quality of stimulus detection. Using a computational approach, we integrated human cellular, synaptic and gene expression data from recent studies to generate detailed models of human cortical L2/3 microcircuits that included the major neuron types with their firing and input integration properties, synaptic models, and connection probabilities. We generated depression microcircuit models by reducing the SST interneuron synaptic and tonic inhibition in the circuit according to gene expression data from recent human studies ${ }^{11}$. We simulated baseline activity and stimulus response as constrained by previous studies of neuron-type activity profiles, to characterize the SNR of cortical processing and the failed/false detection rates of stimuli in health and depression.

\section{Results}

We first generated data driven models of human microcircuits and their baseline and response activity, using human cellular and circuit data whenever available, and data from rodents otherwise (Table S1). The process included modeling single neurons to represent each of the four key neuron types, modeling synaptic properties, and modeling microcircuits with the appropriate proportion of the different neuron types and the connectivity statistics.

\section{Human cortical L2/3 neuron and synaptic models reproduce experimental properties}

We generated single neuron models of the four major cell types in cortical L2/3, using genetic algorithm optimization, to reproduce their electrical properties as measured in human cortical slices. The features of the Pyr neuron model firing in response to depolarizing step currents (e.g. spike rate, height, half-width, adaptation), the frequency-input curve, and sag voltage in response to hyperpolarizing current steps were all within the range (1 - 2 SD) of the experimental population (Fig. 1a, Table S2), except for the after-hyperpolarization depth which was marginal (4 SD). The passive and active firing features of the SST, PV and VIP interneuron models matched the values of the corresponding human neurons (Fig. 1b - d, Tables S3 - S5), and were also within the experimental variance of population data from corresponding neurons in rodents ${ }^{26-28}$. AP halfwidth in the PV models was further from the experimental variance (4.4 - 4.8 SD), which is commonly the case due to the fixed kinetics of the underlying channel models ${ }^{25,29}$. 
a

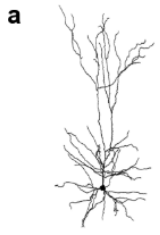

b

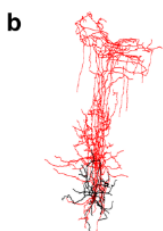

c

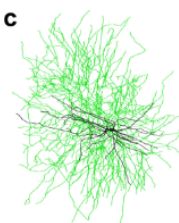

d

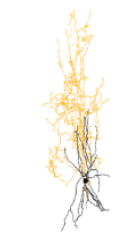

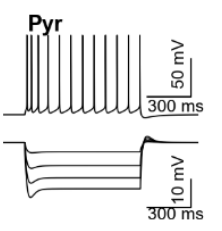
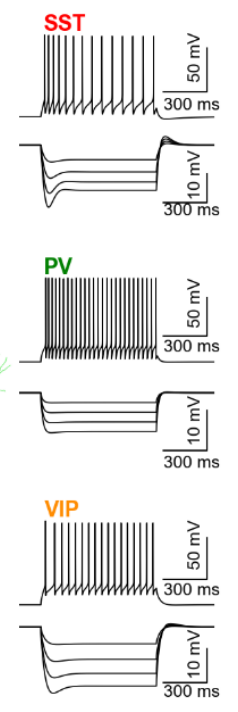
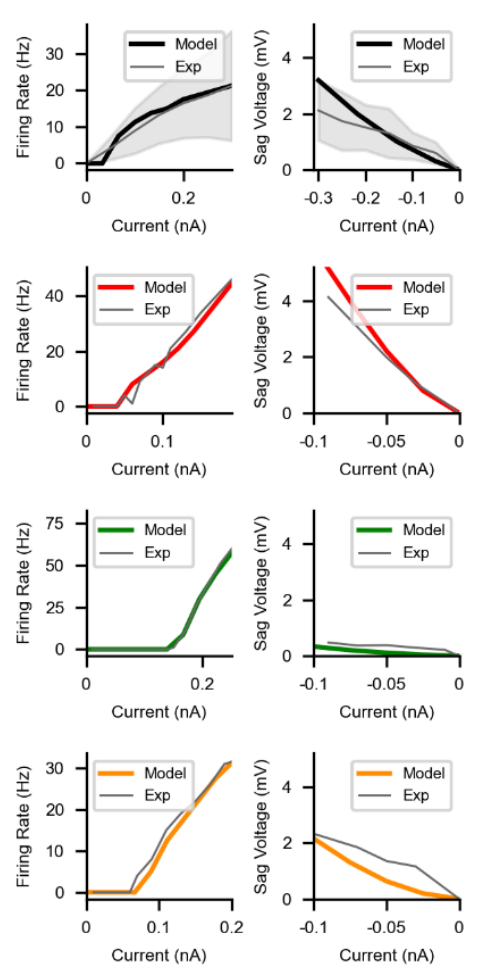

e

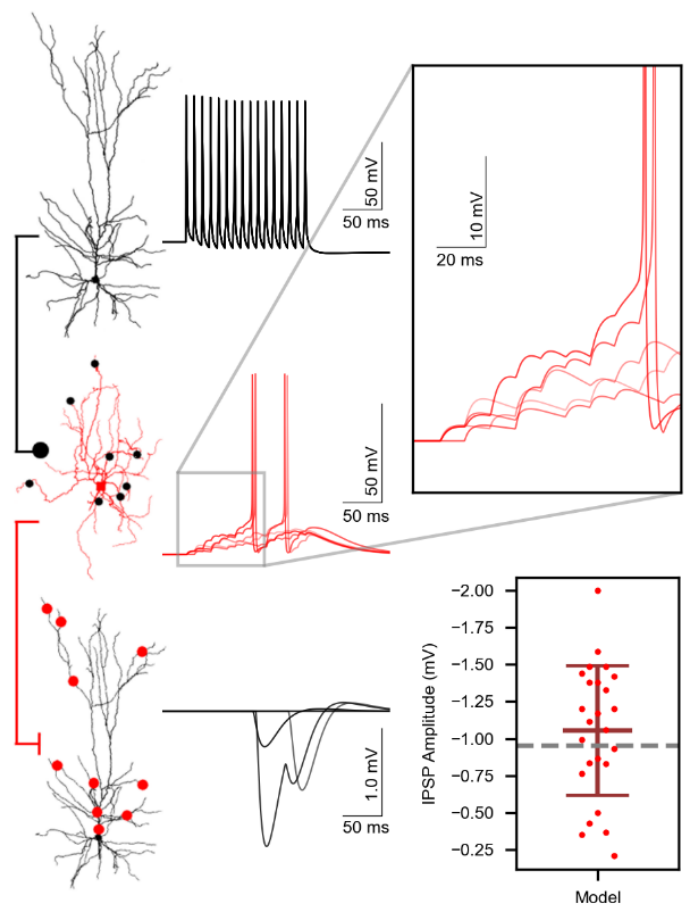

Figure 1. Model human cortical neurons and synaptic connections reproduce experimental properties. a. Left - reconstructed human L2/3 Pyr neuron morphology. Middle - Pyr neuron model response to depolarizing and hyperpolarizing step currents. Right - firing frequency-input curve and sag voltage-input curve of the Pyr neuron model (black) were within the experimental population range (gray, $\mathrm{n}=28$ neurons). b - d. Same as a, but for SST (b), PV (c) and VIP (d) interneurons. Firing frequency-input curve and sag voltage-input curve of the interneuron models (SST, PV, VIP: red, green, orange) matched experimental values of the corresponding single neurons (gray). e. Simulated voltage traces of a human disynaptic inhibition loop model. A Pyr neuron (top) fired 15 spikes at $100 \mathrm{~Hz}$, and the resulting EPSP summation (inset) in an SST interneuron (middle) triggered two spikes, which elicited IPSPs in another Pyr neuron (bottom). The dots on the morphologies indicate synaptic contact locations (black: excitatory; red: inhibitory). The graph on the bottom right shows the simulated IPSP amplitude between SST and Pyr neurons ( $\mathrm{n}=25$ random connections) compared to experimental value (dashed line ${ }^{14}$ ).

Next, we generated models of synaptic connections between the neuron types. We constrained the connections between Pyr neurons and SST interneurons to reproduce properties of the disynaptic inhibition loop as recorded in human brain slices (Fig. 1e). The model synaptic connections from Pyr onto SST interneurons elicited facilitating EPSPs with amplitudes as seen experimentally ${ }^{14}$ (Model: $2.16 \pm 1.35 \mathrm{mV}$, experimental: $2 \mathrm{mV}$ ) and were sufficiently strong to trigger 1 - 2 spikes in the SST interneuron in response to a train of inputs from a single Pyr neuron. The resulting SST IPSP amplitudes in a neighbouring Pyr neuron agreed with the experimental value $^{14}$ (Model: $-1.05 \pm 0.44 \mathrm{mV}$, experimental: $-0.95 \mathrm{mV}$ ). In addition, we constrained the EPSP 
amplitudes between Pyr neurons, and the connections between Pyr and PV neurons to reproduce the experimental values seen in human neurons $(\mathrm{Pyr} \rightarrow \mathrm{Pyr}$ model: $0.42 \pm 0.37 \mathrm{mV}$, experimental: $0.42 \pm 0.45 \mathrm{mV} ; \mathrm{Pyr} \rightarrow \mathrm{PV}$ model: $3.29 \pm 1.16 \mathrm{mV}$, experimental: $3.29 \pm 1.12 \mathrm{mV} ; \mathrm{PV} \rightarrow \mathrm{Pyr}$ model: $-2.23 \pm 1.2 \mathrm{mV}$, experimental: $-2.23 \pm 1.0 \mathrm{mV}$ ). The remaining types of synapses, primarily involving VIP neurons, were constrained using rodent data since human data was unavailable (Table S7). In addition to synaptic inhibition, we modeled tonic inhibition using voltage-clamp recordings of the tonic current in human pyramidal neurons (see Methods). We estimated the tonic conductance using the measured difference between tonic inhibition current and when GABA receptors were blocked. We reproduced the target tonic inhibition current amplitude with a tonic conductance of $0.938 \mathrm{mS} / \mathrm{cm}^{2}$ and applied it to all neurons ${ }^{30}$.

\section{Increased baseline activity (noise) in depression microcircuit models}

We used the neuron and synaptic connection models to simulate human cortical L2/3 microcircuits of 1000 neurons, with the experimental proportions of the different neuron types, their connectivity and column dimensions (Fig. 2a - b). The neurons received random background excitatory input corresponding to cortical and thalamic drive, to enable recurrent activity. We tuned the background excitatory input level and the microcircuit connection probabilities between neuron types to reproduce the baseline firing rates previously reported for the neuron types in vivo (Fig. 2c - d, f). The average firing rate for simulated Pyr neurons was $1.10 \pm 0.05 \mathrm{~Hz}, \mathrm{PV}: 10.31 \pm 0.50 \mathrm{~Hz}, \mathrm{SST}$ : $5.64 \pm 0.18 \mathrm{~Hz}$ and VIP: $3.51 \pm 0.32 \mathrm{~Hz}(\mathrm{n}=10$ microcircuits, rates for non-silent neurons as in experiments, see methods). In addition, we have constrained the microcircuit to reproduce the effects of SST interneuron silencing, which has previously been reported to result in a doubling of baseline Pyr neuron spike rates $^{1}$ (Fig. 2e, $1.10 \pm 0.05 \mathrm{~Hz}$ vs. $2.08 \pm 0.08 \mathrm{~Hz}$, t-test, $p<0.001$ ).

The microcircuit models had an emergent property of oscillatory activity, which was not constrained for explicitly, where population spiking oscillated primarily in the theta $(4-8 \mathrm{~Hz})$ and alpha $(8-12 \mathrm{~Hz})$ frequency bands, and on average exhibited peak frequencies around $10 \mathrm{~Hz}(\mathbf{F i g}$. 2g). The PSD also exhibited a 1/f relationship between power and log frequency (Fig. 2g, inset). These oscillatory properties closely agree with the oscillations seen in human cortical signals invitro and in-vivo ${ }^{31-34}$, providing a validation in support of the models capturing key properties of human cortical L2/3 microcircuits. 

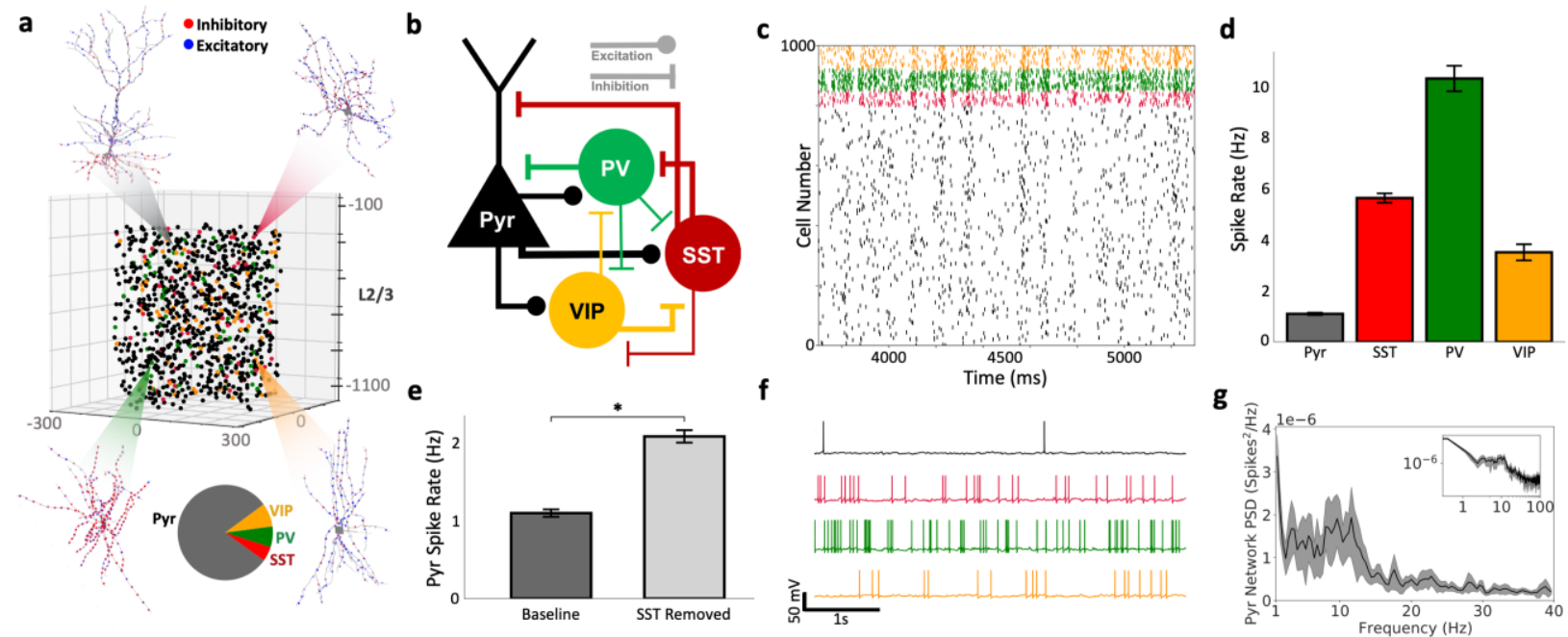

Figure 2. Detailed models of human cortical L2/3 microcircuits reproduce features of intrinsic circuit activity. a) The model microcircuit comprised of 1000 neurons, with the somas distributed in a 500x500x950 $\mu^{3}$ volume along layer $2 / 3$ (250 to $1200 \mu \mathrm{m}$ below pia). The proportions of the different neuron types were based on the experimental literature (pie chart, Pyr: 80\%; SST: 5\%; PV: 7\%; VIP: 8\%). The neurons were modeled with detailed morphologies, as shown in Fig 1, and connected according to the experimental statistics (see Methods). The blue and red dots on each morphology denote example excitatory and inhibitory synapses, respectively. b) The schematic connectivity diagram highlights the key connections between different neuron types in the microcircuit. c) Example raster plot of spiking in different neurons in the microcircuit, color-coded according to neuron type. Neurons received background excitatory inputs to generate intrinsic circuit activity. d) Spike rates in the different neuron types reproduced experimental baseline firing rates (mean and standard deviation, $\mathrm{n}=10$ randomized simulated microcircuits). e) A significant 2 -fold increase in mean Pyr neuron spike rate when SST interneurons were silenced reproduced experimental results ( $\mathrm{n}=10$ microcircuits, paired-sample $\mathrm{t}$-test; $p<0.001)$. f) Example simulated voltage traces for each neuron type. g) Spikes PSD of Pyr neurons, bootstrapped mean and 95\% confidence intervals $(n=10$ randomized microcircuits). Inset - PSD in log scale, illustrating the $1 / \mathrm{f}$ relationship between power and $\log$ frequency.

We next modelled human depression microcircuits by reducing SST interneuron synaptic and tonic inhibition conductance onto the different neuron types by $40 \%$, according to postmortem gene expression data in depression (Fig. 3a - b). We then compared the baseline activity in healthy and depression microcircuits, across all neurons of each type. The average baseline firing rate of Pyr neurons was significantly higher in depression microcircuits compared to healthy microcircuits (Fig. 3c - d, healthy: $0.77 \pm 0.05 \mathrm{~Hz}$, depression: $1.20 \pm 0.07 \mathrm{~Hz}, \mathrm{n}=200$ randomized microcircuits, t-test $p<0.05$, Cohen's $d=7.06$ ). Next, we quantified the effect of different levels of SST interneuron inhibition reduction on baseline rates of Pyr neurons by simulating microcircuits with 0 - 100\% reduction compared to the healthy level. The baseline firing of Pyr neurons increased approximately linearly with reduced SST interneuron inhibition (Fig. 3d). Similar increases in firing rates were observed in the interneuron populations of the depression 
microcircuits compared to healthy microcircuits (Fig. 3e, SST: $5.62 \pm 0.27$ vs. $7.53 \pm 0.39 \mathrm{~Hz}$; PV: $10.19 \pm 0.51$ vs. $15.99 \pm 0.54 \mathrm{~Hz}$; VIP: $3.52 \pm 0.37$ vs. $7.26 \pm 0.49 \mathrm{~Hz}, \mathrm{n}=200, p<0.05$ for all). The higher activity in depression microcircuits when no stimulus was given therefore indicated an increased noise level with respect to processing incoming stimuli.
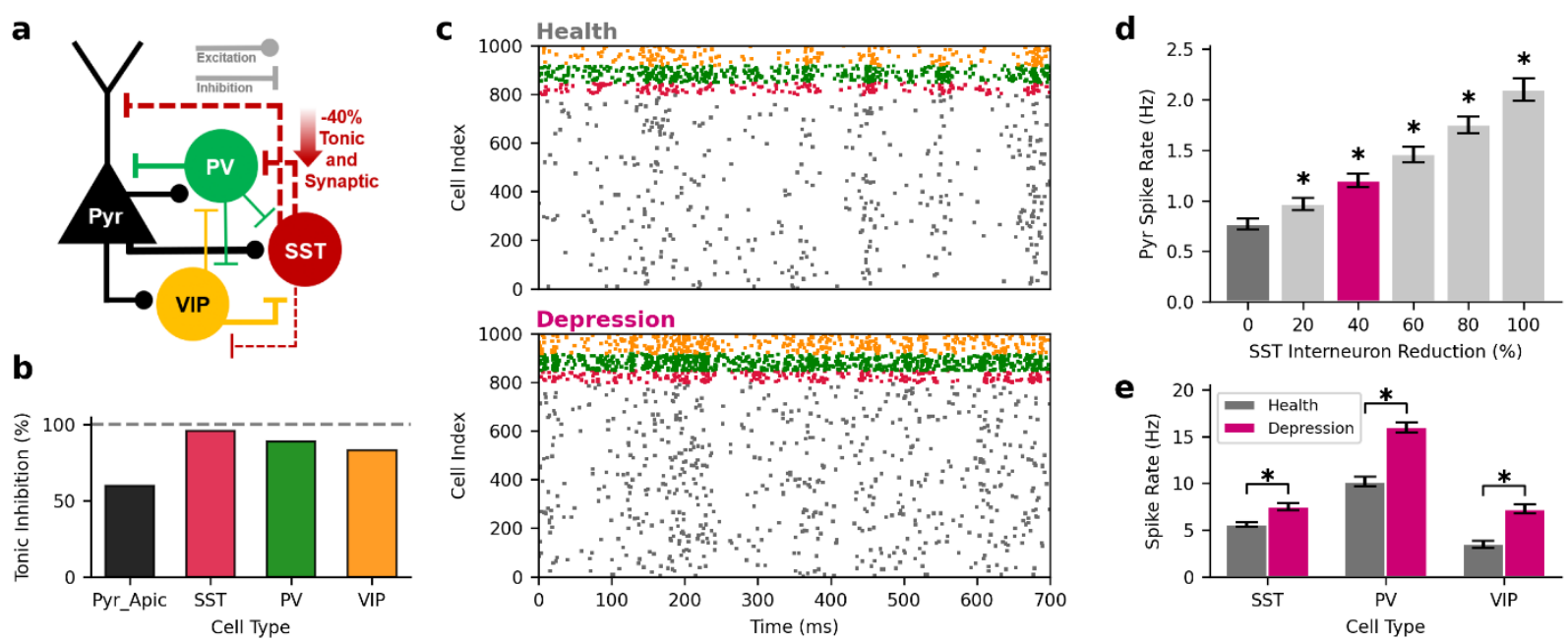

Figure 3. Increased baseline activity (noise) in depression microcircuit models. a. Depression microcircuits were modeled according to gene expression data, with $-40 \%$ synaptic and tonic inhibition conductance from SST interneurons onto the other neuron types. b. Relative tonic inhibition conductance onto the different neuron types in depression microcircuits, compared to healthy microcircuits (dashed line). c. Example raster plot of simulated baseline spiking in a healthy microcircuit model (top) and depression microcircuit model (bottom). d. Increased intrinsic Pyr neuron firing in depression microcircuit models (n $=200$ randomized microcircuits per condition, purple, $p<0.05$, Cohen's $d=7.06$, healthy level in dark grey). The relationship between SST interneuron inhibition reduction and baseline Pyr neuron firing rate was approximately linear. e. Increased interneuron baseline firing rates in depression microcircuit models $(p<0.05)$.

\section{Reduced SNR of cortical processing in human depression microcircuits}

To better understand the implications of the increased baseline firing in depression microcircuits, we compared their evoked response activity to that of healthy microcircuits. We modelled healthy evoked response to a brief stimulus by reproducing the temporal profile and average firing rates in the neuron types as measured in cortical layer $2 / 3$ in vivo (in rodents). VIP and PV interneurons were stimulated earliest and consequently silenced SST interneurons. Pyr neurons were stimulated shortly after and responded with a brief peak firing followed by a sustained lower response rate, although still above baseline, that lasted $\sim 100 \mathrm{~ms}$ (Fig. 4a, b). Over the 5 - $55 \mathrm{~ms}$ window post stimulus, Pyr neurons fired at $2.49 \pm 0.61 \mathrm{~Hz}$ on average $(\mathrm{n}=200$ randomized microcircuits $)$. We then applied this stimulus paradigm to the depression microcircuits and found no significant 
change in average Pyr response rate (Fig. 4c, $2.52 \pm 0.47 \mathrm{~Hz}$, Cohen's $d=0.06$ ). The evoked response of interneurons was similar between healthy and depression microcircuits as well (Fig. 4i, SST: $2.74 \pm 1.34$ vs. $3.21 \pm 1.55 \mathrm{~Hz}$, Cohen's $d=0.32$; PV: $30.62 \pm 4.79$ vs. $32.06 \pm 3.43 \mathrm{~Hz}$, $d=0.35 ;$ VIP: $32.88 \pm 2.92$ vs. $34.94 \pm 2.52 \mathrm{~Hz}, d=0.76)$.

a
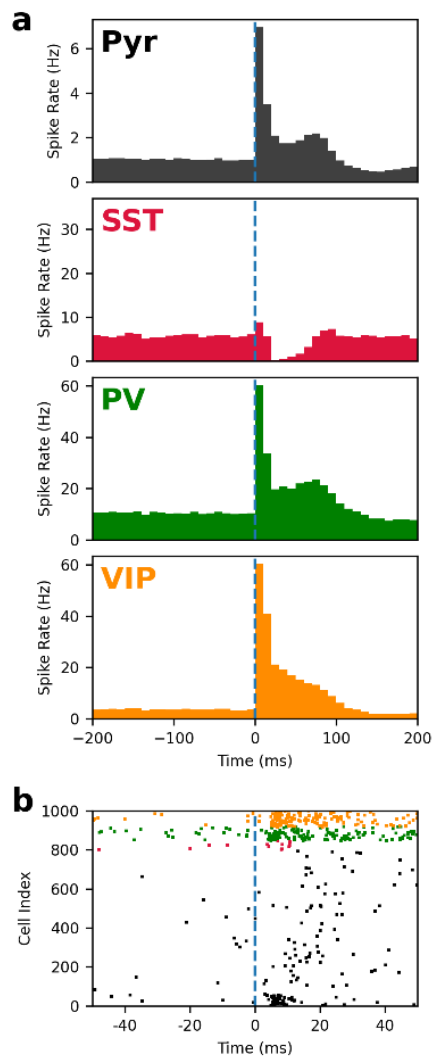
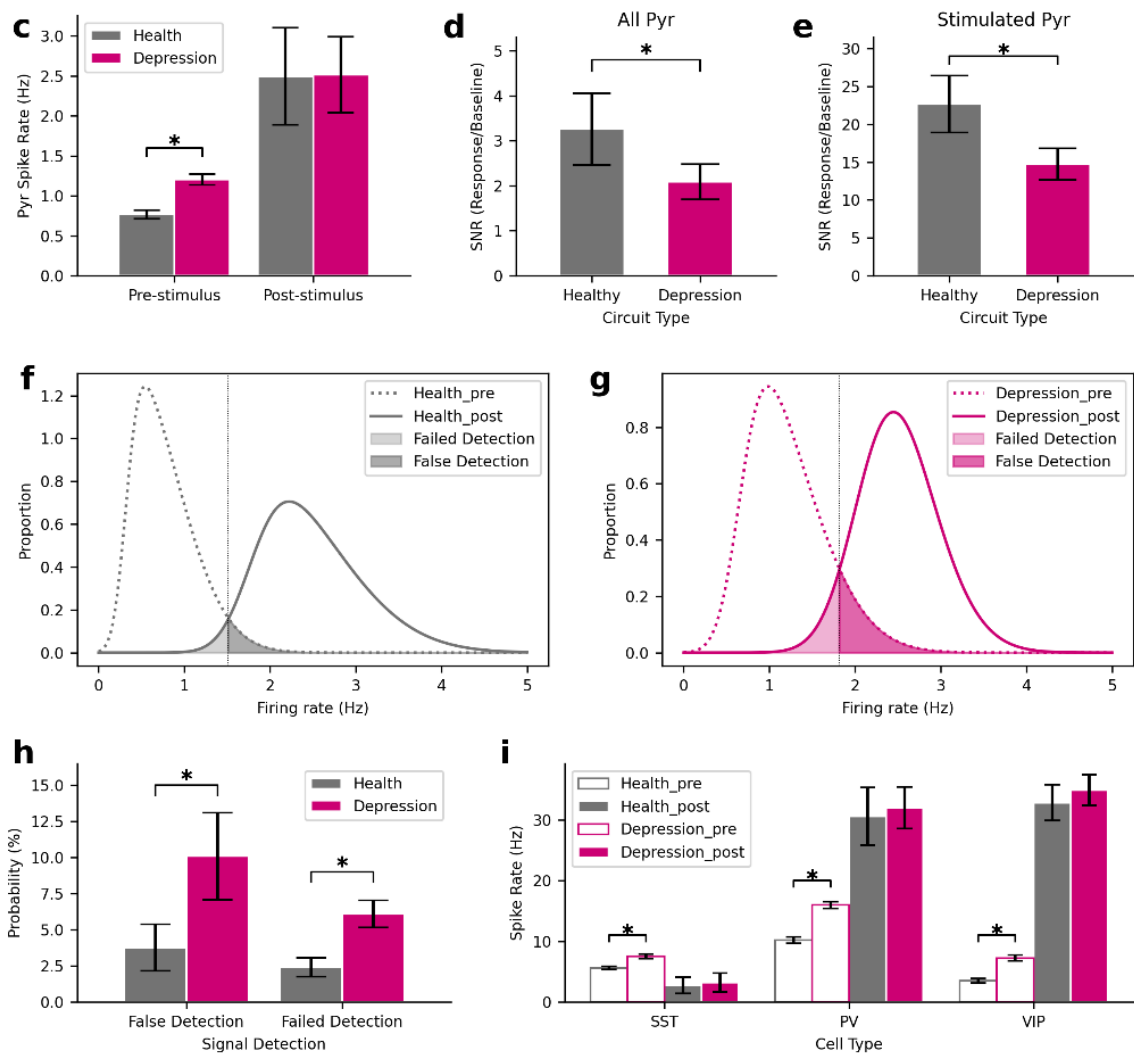

i
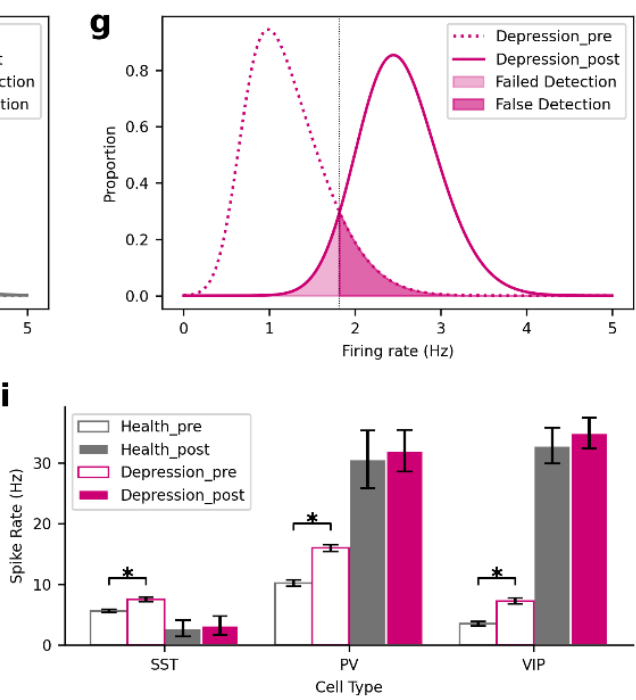

Figure 4. Decreased cortical SNR and impaired stimulus detection in depression microcircuit models. a. Average peristimulus time histogram ( $\mathrm{n}=200$ randomized microcircuits) of simulated response to thalamic stimulus in healthy microcircuits reproduce response firing rates and profiles recorded in the different neuron types in awake rodents. Dashed line denotes the stimulus time. b. Example raster plot of simulated spike response in a healthy microcircuit model. c. Baseline Pyr neuron firing rates in depression microcircuits were significantly higher than in healthy microcircuits $(p<0.05$, Cohen's $d=7.06)$, but response rates were similar (Cohen's $d=0.06$ ). d. Decreased SNR (ratio of response vs. baseline Pyr neuron firing rates) in depression microcircuits $(p<0.05)$. e. Same as d, but for the stimulated Pyr neurons only. f. Distribution of pre- and post- stimulus firing rates in $50 \mathrm{~ms}$ windows in healthy microcircuits $(\mathrm{n}=1950$ windows $\mathrm{x} 200$ microcircuits pre-stimulus, $\mathrm{n}=50$ windows post-stimulus). The vertical dotted line denotes the decision threshold of signal detection (see Methods). g. Same as f, but for depression microcircuits. $\mathbf{h}$. Increased probability of false detection and failed detection in depression vs. healthy microcircuits. i. Baseline and response firing rates in interneurons in healthy and depression microcircuits.

We calculated the overall SNR in the healthy and depression microcircuits, as the ratio of the response activity (signal) and baseline activity (noise) across the Pyr neuronal population, and 
found significantly $(>50 \%)$ reduced SNR in depression microcircuits (Fig. 4d, health: $3.26 \pm 0.80$, depression: $2.09 \pm 0.39, p<0.05)$. The reduction in SNR was similarly significant when examining only the stimulated Pyr neurons (Fig. 4e, health: $22.67 \pm 3.78$, depression: $14.75 \pm 2.10, p<0.05$ ). To further investigate the effect of reduced SNR in cortical processing, we determined the corresponding change in false/failed signal detection rates. We calculated the distribution of spike rates of all Pyr neurons in $50 \mathrm{~ms}$ windows pre and post stimulus to calculate the probability of false positive/negative errors in stimulus processing (Fig. $\mathbf{4 f}, \mathbf{g}$ ). We found a significant increase, more than doubling, in the false detection rates (health: $3.76 \pm 1.60 \%$, depression: $10.07 \pm 3.02 \%$, $p<0.05$ ) and the failed detection rates (health: $2.40 \pm 0.67 \%$, depression: $6.01 \pm 0.94 \%, p<0.05$ ) in depression (Fig. 4h).

To systematically assess the impact of different levels of SST interneuron inhibition reduction, we simulated microcircuits with 0 - 100\% reduction compared to the healthy level. SNR decreased with the level of SST inhibition reduction (Fig. 5a, 0\%: $3.26 \pm 0.80,40 \%: 2.09 \pm 0.39$, $100 \%: 1.30 \pm 0.27$ ), with a sharper decrease up to the reduction level estimated in depression (40\%), and a more moderate change beyond that level. Interestingly, the rate of false signal detection increased nonlinearly with the level of inhibition reduction (Fig. 5b). There was an effect threshold around the level of reduction estimated in depression, with a negligible change for a smaller reduction $(0 \%: 3.76 \pm 1.60 \% ; 20 \%: 4.94 \pm 1.95 \%$, Cohen's $d=0.66)$ and a sharper increase at the depression level and beyond (40\%: $10.07 \pm 3.02 \%, p<0.05$, Cohen's $d=2.61$; $100 \%: 40.07 \pm 6.70 \%, p<0.05$, Cohen's $d=7.45$ ). Similar nonlinear increase and threshold effect were observed for failed detection rates (Fig. 5c; 0\%: $2.40 \pm 0.67 \%$; 20\%: $2.21 \pm 0.61 \%$, Cohen's $d=-0.30 ; 40 \%: 6.09 \pm 0.94 \%, p<0.05$, Cohen's $d=4.5 ; 100 \%: 15.44 \pm 3.27 \%, p<0.05$, Cohen's $d=5.5)$.

a

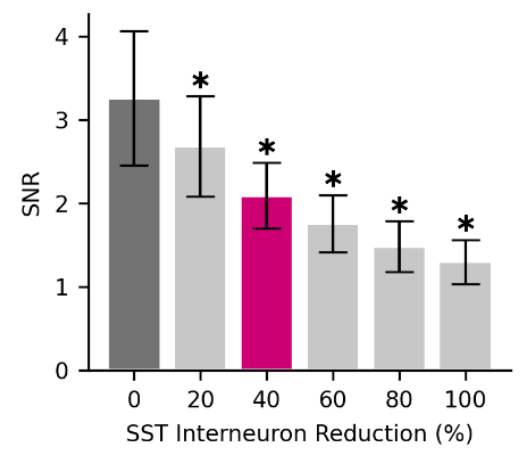

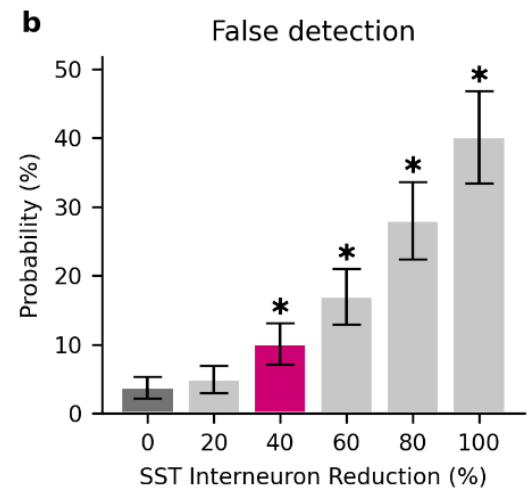

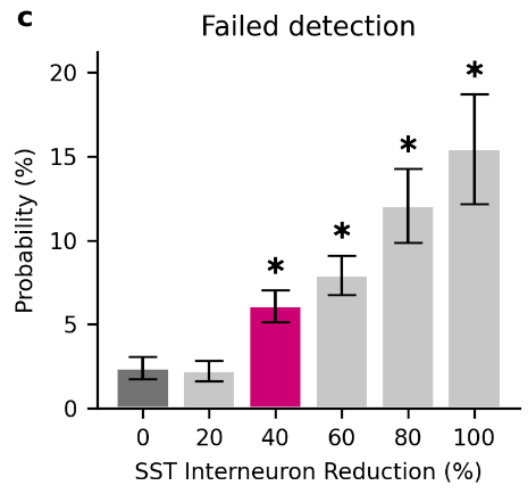


Figure 5. Effect of different levels of reduced SST interneuron inhibition on SNR and signal detection. a-c. SNR(a), false signal detection rates(b) and failed signal detection rates(c) at different levels of SST interneuron inhibition reduction.

\section{Discussion}

This study determined the implications of reduced inhibition in depression on cortical processing in microcircuits, using novel detailed models of human cortical L2/3 microcircuits that integrated human cellular, circuit, and gene-expression data in health and depression. Our simulations of microcircuit response and baseline activity showed that the reduced SST interneuron inhibition in depression estimated by gene expression resulted in a significantly decreased SNR of cortical processing and increased failed/false signal detection rates. Our results thus examined and provided a mechanistic validation for a leading hypothesis of reduced SST inhibition as a pathological mechanism for dysfunctional processing in depression. This lends support for establishing SST inhibition as a target mechanism for new pharmacology in treating depression. Furthermore, our results make testable quantitative predictions about the correspondence between different degrees of reduced SST inhibition and the resulting cognitive deficits that are relevant to symptoms such as rumination and impaired signal detection, which may serve to improve subtyping of depression for better diagnosis, treatment, and drug monitoring in the future.

The reduced SNR and signal detection quality that we found in the depression microcircuit models may underlie the inability to break internal loops of thought in rumination symptoms ${ }^{3}$, and the impaired signal detection that results in suboptimal decisions in depression ${ }^{35,36}$. The increase of failed detection in our depression microcircuit models was of a similar level to previous studies ${ }^{36}$, and these studies similarly found a larger increase in false detection rate compared to failed detection rate in depression. The relationship between dysfunctional microcircuit processing and reduction of SST interneuron inhibition that we quantified may serve as biomarkers for diagnosing depression type and severity, and for informing on the required drug dose with positive-allostericmodulation of $\alpha 5-\mathrm{GABA}_{\mathrm{A}}$ receptors ${ }^{12,37}$. Future studies should examine how the relationship between reduced SST inhibition and processing is further affected by a disruption in neuromodulation by serotonin and acetylcholine, which is implicated in depression ${ }^{38-40}$.

We have applied gene expression data to estimate a reduction in the strength (conductance) of inhibitory connections from SST interneurons ${ }^{11}$. While the abnormal gene expression may 
alternatively reflect a reduced number of $\operatorname{synapses}^{41}$, the net inhibition decrease in either case would be largely similar. A comparison between conductance decrease and synaptic loss will be interesting to investigate in future studies. Our models of depression focused on the implication of reduced inhibition at the microcircuit scale, but future studies could explore the implications on multiregional processing in depression, by modelling and stimulating several detailed microcircuits corresponding to the different relevant brain areas and their interactions ${ }^{42}$. Multiregional simulations will help distinguish the similarities and differences of SST inhibition effects in various neurological disorders where these interneurons are implicated ${ }^{9,16}$. Multiregional processing simulations will also enable examining the implications on the later stages of the cortical response, where SST interneurons may provide feedback inhibition to reduce excitation when stimuli are similar to contextual surroundings ${ }^{43}$, or serve to shorten response duration ${ }^{44}$. Pathologically reduced inhibition in such cases may result in prolonged response time and thus slower processing of subsequent stimuli ${ }^{37}$.

We developed novel models of human cortical microcircuits to study depression in the context of the human cortex. We were able to improve upon existing models of human L2/3 neurons to better reproduce the firing frequency-input relationship and the sag voltage, thus capturing important input integration properties of neurons within the microcircuit. Further improvements to our models can include dendritic ion-channels mediating backpropagating action potentials and calcium spikes that have been well characterized in rodents ${ }^{29}$. These dendritic properties still remain to be better characterized in humans, but recent studies suggest that calcium spikes may play a role in mediating nonlinear processing in human Pyr neurons ${ }^{22}$. We note, however, that our microcircuit models are still capable of NMDA nonlinearities, which play an important role in the cortical microcircuit ${ }^{45}$. A further refinement of the models would be constraining the connectivity between neuron types in the human cortex, which is largely unavailable at present but is expected to become available in the near future ${ }^{46}$. Nevertheless, the model spike oscillations in theta and alpha frequency bands, and the 1/f relationship between power and $\log$ frequency agree with the oscillations seen in human cortical signals experimentally ${ }^{31-34}$ and serve as a validation in support of the models capturing key properties of human cortical L2/3 microcircuits. As human data from other cortical layers becomes more available, future directions should examine the implication of reduced SST inhibition in depression on signal propagation across layers ${ }^{47}$. We examined the effect of SST inhibition on cortical 
processing using the response rate profile in the different neuron types based on available somatosensory studies in rodents, due to the lack of similar data in humans. The relevance of rodent response data is supported by the important similarities between rodent and human cortex in terms of ion-channel mechanisms, neuron types, and microcircuit connection motifs such as the SST inhibitory disynaptic loop ${ }^{14,15,48}$. Future studies should investigate how the effects we found differ in rodent microcircuit models, with smaller neurons and weaker synapses, to better establish the preclinical relevance of the rodent animal model of depression. Due to the above-mentioned species similarities in neuron types and circuit motifs we expect the results to largely hold for rodent microcircuits as well, although with possible nuanced differences in the extent and magnitude of the effects. Our detailed models of healthy microcircuits can further serve to study the cellular and circuit mechanisms of other neurological disorders such as schizophrenia and epilepsy. Furthermore, our models are implemented in $\mathrm{LFPy}^{49}$ and thus can be used to study the associated electroencephalography signals and local field potentials from our depression microcircuit models to identify biomarkers for improving diagnosis and monitoring ${ }^{50}$. All models and simulation code will be available openly online upon publication.

\section{Methods}

Experimental Data. We used whole-cell recordings from human medial temporal gyrus, layer 2/3 (L2/3) Pyr neurons ${ }^{24}(\mathrm{n}=28$ neurons, 9 neurons from 3 male subjects and 19 neurons from 5 female subjects), and from human putative SST (Neuron ID: 571700636), PV (Neuron ID: 529807751) and VIP (Neuron ID: 525018757) interneurons available from the Allen Brain Atlas $^{51,52}$. We used reconstructed human neuron morphologies from the Allen Brain Atlas for a Pyr neuron (Neuron ID: 531526539) and the above three interneurons. We used five hyperpolarizing and depolarizing current steps from the electrophysiological data for model optimizations (Tables S2 - S5). Three depolarizing supra-threshold current steps were used for targeting appropriate firing features at low, medium and high firing rates. A small hyperpolarizing step current was used for fitting passive features, and a large hyperpolarizing current step was used for fitting sag voltage.

In addition, we used current recordings of tonic inhibition in human cortical L2/3 Pyr neurons (10 cells: 9 cells from 3 male subjects, 1 cell from 1 female subject). Written informed consent was obtained from all participants, in accordance with the Declaration of Helsinki and the 
University Health Network Research Ethics board. The data was collected using surgery resection, solutions, tissue preparation, and recording equipment described previously ${ }^{24}$. For voltage-clamp recordings of tonic current, low-resistance patch pipettes (2-4 M $\Omega$ ) were filled with a CsCl-based solution containing (in mM) $140 \mathrm{CsCl}, 10$ EGTA, 10 Hepes, 2 MgCl2, 2 Na2ATP, 0.3 GTP, and 5 QX314 adjusted to $\mathrm{pH} 7.3$ with $\mathrm{CsOH}$. Experiments were performed with excitatory (APV 25 $\mu \mathrm{M}$, Sigma; CNQX $10 \mu \mathrm{M}$, Sigma) and GABA (CGP-35348 $10 \mu \mathrm{M}$, Sigma) synaptic activity blocked as in previous studies ${ }^{53,54}$. The junction potential was calculated to be $4.3 \mathrm{mV}$ and the holding potential was $-74.3 \mathrm{mV}$ after junction potential correction. Mean amplitude of baseline tonic current in presence of GABA $(5 \mu \mathrm{M})$ and glutamatergic blocker was $65.8 \pm 10.2 \mathrm{pA}(\mathrm{n}=10$ cells).

Human L2/3 Microcircuit Models. We simulated cortical L2/3 microcircuits comprised of 1000 neurons distributed along a 500x500x950 $\mu^{3}$ volume (250 to $1200 \mu \mathrm{m}$ below pia, spanning $\mathrm{L}_{2} / 3^{21}$ ) using NEURON ${ }^{55}$ and $\mathrm{LFPy}^{49}$. We used human data to constrain the models where available (Table S1 and see below). Neurons of a given type had the same model (morphology and biophysical properties, see below) but differed in synaptic connectivity and background input (see below). The proportions of the four neuron types in the microcircuit were: $80 \% \mathrm{Pyr}, 5 \%$ SST, $7 \% \mathrm{PV}$, and $8 \%$ VIP, according to the relative neuron densities found in the rodent and human cortical L2/3 in previous studies ${ }^{56,57}$, and RNA-seq data from the Allen Human Brain Atlas ${ }^{17,52}$.

Neuron Models. We derived multi-compartmental conductance-based models for the different neuron types using multi-objective optimization with a genetic algorithm from previous in-house $\operatorname{code}^{29}$ for optimizing the Pyr and SST neuron models, and the BluePyOpt Python module ${ }^{58}$ for optimizing the PV and VIP neuron models. We used a set of ion channel mechanisms taken unchanged from previously published models ${ }^{29,59}$. Pyr and SST neuron models were fitted using a two-step process, where first h-current and passive parameters $\left(\bar{g}_{H}, \bar{g}_{\text {Leak }},\right)$ were fitted to capture passive and sag voltage features during hyperpolarizing current steps, and then other ion channel parameters $\left(\bar{g}_{N a}, \bar{g}_{K}, \bar{g}_{C a}\right)$ were fitted to capture spiking features during depolarizing current steps (Tables S2 - S3). The PV and VIP neuron models were fitted in one step where passive and firing features were optimized simultaneously (Tables S4 - S5). For all models, $R_{a}=100 \Omega \mathrm{cm}, E_{N a}=$ $50 \mathrm{mV}, E_{K}=-85 \mathrm{mV}$, and CaDynamics gamma $=0.0005^{29}$. Axonal $\mathrm{Na}_{\mathrm{T}}$ kinetics parameters were 


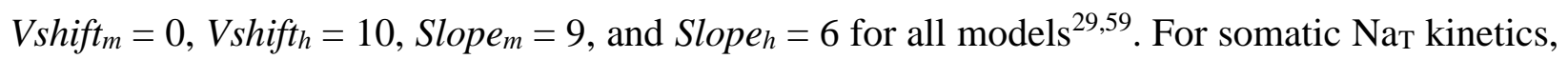
Vshift $_{m}=13$, Vshift $_{h}=15$, Slope $_{m}=7$, and Slope ${ }_{h}=6^{29,59}$, except for the PV neuron model where these parameters at the soma were the same as in the axon to compensate for reduced action potential propagation brought on by the axon replacement method. The specific membrane capacitance $\left(c_{m}\right)$ was $1 \mu \mathrm{F} / \mathrm{cm}^{2}$, and $2 \mu \mathrm{F} / \mathrm{cm}^{2}$ in the dendrites to compensate for spine area loss in Pyr neuron reconstructions ${ }^{60,61}$ or where required to reproduce membrane time constants in interneurons, possibly due to errors in dendritic diameter estimation (Table S6). $\bar{g}_{H}$ was distributed uniformly across all dendritic sections, with the exception of Pyr apical dendrites which followed an exponential increase with distance from soma as follows:

$$
\bar{g}_{H}=\bar{g}_{H, \text { soma }}\left(-0.8696+2.0870 \exp \left(3.6161\left(X_{\text {relative }}\right)\right)\right)
$$

where $X_{\text {relative }}$ is the relative distance from soma to maximal length (i.e. from 0 to 1$)^{29}$. Model optimization was run using parallel computing clusters $\left(\operatorname{SciNet}^{62,63}\right.$ : 400 processors with a population size of 1000, for 1000 generations and an approximate total runtime of 2 hours; Neuroscience Gateway, NSG ${ }^{64}: 400$ processors with a population size of 400, for 300 generations and an approximate total runtime of 5 hours). Model performance in reproducing the electrophysiological features was assessed in terms of standard deviation from the experimental mean. For Pyr neuron models we used the statistics over the set of recorded neurons (see above). For the interneurons, where only a single human neuron was clearly identified for each neuron type, we used the values of the human neuron with population variance from the rodent literature ${ }^{26-}$ 28.

Synaptic Connectivity Models. Where possible, we constrained synaptic parameters using the human experimental literature. Otherwise, we used parameters from the rodent literature (the Blue Brain Project ${ }^{65}$ ). We used previous models of NMDA/AMPA excitatory and GABAA inhibitory synapses, that incorporated presynaptic short-term plasticity parameters for vesicle-usage, facilitation, and depression, as well as separate time constant parameters for the AMPA and NMDA components of excitatory synapses ${ }^{45,50,66}$. We set the same time constant parameters for all connection types $\left(\tau_{\text {rise }, N M D A}=2 \mathrm{~ms} ; \tau_{\text {decay }, N M D A}=65 \mathrm{~ms} ; \tau_{\text {rise }, A M P A}=0.3 \mathrm{~ms} ; \tau_{\text {decay }, A M P A}=3 \mathrm{~ms}\right.$; $\left.\tau_{\text {rise }, G A B A}=1 \mathrm{~ms} ; \tau_{\text {decay }, G A B A}=10 \mathrm{~ms}\right)$, as well as the reversal potential values $\left(E_{\text {exc }}=0 \mathrm{mV} ; E_{\text {inh }}=\right.$ $80 \mathrm{mV}$ ). 
We fitted the synaptic conductance, and vesicle usage parameters ${ }^{45}$ where available, using the human experimental literature for most of the key connection types: $\mathrm{Pyr} \rightarrow \mathrm{Pyr}$ connections ${ }^{20}$, $\mathrm{Pyr} \rightarrow \mathrm{SST}$ connections and $\mathrm{SST} \rightarrow \mathrm{Pyr}$ connections ${ }^{14}, \mathrm{PV} \rightarrow \mathrm{Pyr}$ connections ${ }^{19}$, and $\mathrm{Pyr} \rightarrow \mathrm{PV}$ connections ${ }^{67}$. We simulated the experimental conditions (e.g., chloride reversal potential, and holding currents) and adjusted the conductance and vesicle usage parameters to achieve the target postsynaptic amplitudes and failure rates on average across 200 randomizations of synaptic locations and events. For the conductance and vesicle usage parameters of all other connection types, as well as the depression, facilitation, and numbers of synaptic contacts for all connections, we used model parameters reported for rodents by the Blue Brain Project ${ }^{65,68}$. The synaptic parameters of the different connections are summarized in Table S7. Synaptic locations onto Pyr neurons were random but depended on the connection type, where $\mathrm{Pyr} \rightarrow \mathrm{Pyr}$ synapses were placed on both basal and apical dendritic compartments, PV $\rightarrow$ Pyr connections were placed on basal dendritic compartments, and $\mathrm{SST} \rightarrow \mathrm{Pyr}$ connections were placed on apical dendritic compartments. Apart from these specifications, synapse locations were chosen randomly from a uniform distribution.

We set unidirectional connection probability $\left(p_{c o n}\right)$ according to the rodent literature (Blue Brain Project and Allen Brain Institute), except for $\mathrm{Pyr} \rightarrow \mathrm{Pyr}$ connections, where $p_{\text {con }}$ was set to $15 \%$ according to the human literature ${ }^{20}$. We adjusted some connection probabilities guided by the reported experimental ranges to reproduce the intrinsic activity in the microcircuit (see below). The connection probabilities in the microcircuit are summarized in Table S7.

Tonic Inhibition Models. We modelled tonic inhibition using a model for outward rectifying tonic inhibition ${ }^{69}$ and estimated the conductance of tonic inhibition $\left(G_{t o n i c}\right.$; uniformly across all somatic, basal, and apical compartments) using current magnitude in human cortical L2/3 Pyr neurons (see above), which agreed with values from the human L5/6 literature ${ }^{30}$. We replicated the experimental conditions by setting the GABA reversal potential to $-5 \mathrm{mV}$ (i.e. consistent with a high chloride intracellular solution), setting the holding potential to $-75 \mathrm{mV}$ in voltage-clamp mode, and reproduced the target tonic inhibition current amplitude with $\mathrm{G}_{\text {tonic }}: 0.938 \mathrm{mS} / \mathrm{cm}^{2}$. We used the same value for the interneurons since the total tonic inhibition current recorded in interneurons was similar to that of Pyr neurons after correcting for cell capacitance ${ }^{30}$. 
Modelling Microcircuit Baseline and Response Activity. We constrained the microcircuit to generate spike rates at baseline and during response (see below) as reported for the different neuron types in vivo. For baseline activity of Pyr neurons, we used values recorded in humans and monkeys in vivo ${ }^{70}$ (Subject 1: $0.66 \pm 0.51 \mathrm{~Hz}$; Subject 2: $0.32 \pm 0.38 \mathrm{~Hz}$; Monkey: $1.31 \pm 1.11$ $\mathrm{Hz}$ ), and for baseline activity of the interneuron types we used values recorded in rodents in vivo $(\mathrm{SST}: 6.3 \pm 0.6 \mathrm{~Hz} \text {; PV: } 9.4 \pm 2.1 \mathrm{~Hz} \text {; VIP: } 3.7 \pm 0.7 \mathrm{~Hz})^{1,44}$. We note that baseline Pyr rates in human and rodents in the above studies were largely similar. We reproduced the baseline firing rates by adjusting the connection probability values guided by the reported experimental ranges (see above) for all connection types except $\mathrm{Pyr} \rightarrow \mathrm{Pyr}$ connections, and by adjusting the background input (see below). We calculated the simulated rates across non-silent neurons $(>0.2 \mathrm{~Hz})$, as in the above experimental studies. We further constrained the microcircuit model to reproduce a doubling of baseline Pyr neuron spike rates when SST interneurons were silenced, as seen experimentally $^{1}$ (baseline: $1.2 \pm 0.2 \mathrm{~Hz}$; SST silenced: $2.2 \pm 0.3 \mathrm{~Hz}$ ).

The microcircuit received random background excitatory input using Ornstein-Uhlenbeck (OU) point processes ${ }^{71}$, placed at halfway the length of each dendritic arbor to ensure similar levels of inputs along each dendritic path to the soma. For the Pyr neuron models, we placed 5 additional OU processes along the apical trunk at 10\%,30\%,50\%, 70\%, and 90\% of the apical dendritic length. We set the base excitatory OU conductances to the following: Pyr = $28 \mathrm{pS}$; SST $=30 \mathrm{pS}$; $\mathrm{PV}=280 \mathrm{pS} ; \mathrm{VIP}=66 \mathrm{pS}$. We set the inhibitory OU conductance to 0 , since the model microcircuit provided sufficient inhibition. Furthermore, we scaled the OU conductance values to increase with distance from soma by multiplying them with the exponent of the relative distance from soma (ranging from 0 to 1$): \bar{g}_{O U}=\bar{g} \times \exp \left(X_{\text {relative }}\right)$.

We modelled the spike response rates and profiles using values recorded in the different neuron types in vivo, in behaving rodents ${ }^{1}$ (change in firing rate compared to pre-stimulus rate: Pyr $2.2 \pm 1.0 \mathrm{~Hz}$, SST: $-2.9 \pm 0.9 \mathrm{~Hz}$; PV: $21.8 \pm 8.9 \mathrm{~Hz}$; VIP: $14.0 \pm 3.0 \mathrm{~Hz}$ ). We reproduced the response rates by synaptic stimulation of the model microcircuits representing a bottom-up (thalamic) input. We tuned the microcircuit connectivity, the stimulus input conductance and stimulus timing in the different neuron types to reproduce the experimental response profiles and timing of activation ${ }^{1,44}$. The model neurons were stimulated using excitatory AMPA/NMDA synapses with the same synaptic dynamics and number of contacts as the cortical excitatory synapses above. 55 Pyr neurons were stimulated in the basal dendrites, with $2-4$ ms delay post- 
stimulus and a conductance of $4 \mathrm{nS}$. PV interneurons were stimulated in two groups and phases: early (35 PV interneurons, delay $=2-2.5 \mathrm{~ms}$, conductance $=2 \mathrm{nS}$ ) and late (70 PV interneurons, delay $=9-13 \mathrm{~ms}$, conductance $=4.2 \mathrm{nS}$ ). VIP interneurons were similarly stimulated in two groups and phases: early (15 VIP interneurons, delay $=0.5-4.5 \mathrm{~ms}$, conductance $=2.8 \mathrm{nS})$ and late $(65$ VIP interneurons, delay $=7-12 \mathrm{~ms}$, conductance $=2.2 \mathrm{nS}$ ). This stimulus paradigm was applied to 200 randomized healthy and depression microcircuits. Average pre-stimulus rates were calculated over the 2 seconds before stimulus onset. Average post-stimulus rates were calculated over the 5 - $55 \mathrm{~ms}$ window after stimulus onset. To examine and visualize the stimulus response in each of the four cell types, we calculated peristimulus time histograms (PSTH) of the spike counts of non-silent cells (>0.2 Hz) in $10 \mathrm{~ms}$ bins, $200 \mathrm{~ms}$ before and after stimulus, pooled across 200 randomized microcircuits.

Spikes PSD. Power spectral density (PSD) of Pyr neuron population spiking during baseline microcircuit activity was computed by first converting the spike times into binary spike train vectors and then summing the binary spike train vectors across all Pyr neurons. PSD was then computed from the summed spike train vectors using Welch's method ${ }^{72,73}$ from the SciPy python module (nperseg=100,000 sampling points, equivalent to $2.5 \mathrm{~s}$ time windows). PSD vectors across random seeds were then bootstrapped at each frequency 500 times, from which the resulting bootstrapped means and $95 \%$ confidence intervals were computed.

Human Depression Microcircuit Models. We modelled depression microcircuit models by reducing the conductance of SST interneuron synaptic and tonic inhibition on all cell types by $40 \%$, as indicated by the $40 \%$ reduction of SST expression in SST interneurons in L2/3 in postmortem brain tissue of patients with depression ${ }^{11}$. For Pyr neurons, we selectively decreased the tonic inhibition by $40 \%$ in the apical dendrites. For each interneuron, we estimated the relative contribution of SST interneurons to the total inhibitory input and reduced the tonic inhibition by $40 \%$ of the relative contribution. This relative contribution was calculated by multiplying the SST synaptic conductance, connection probability, and the number of contacts, and dividing by the summed contributions of all interneuron types. 
False/Failed Signal Detection Rates. We calculated the error rates in stimulus processing by first computing the distribution of pre-stimulus firing rates of all Pyr neurons per circuit run (sliding windows of $50 \mathrm{~ms}$ over 2 seconds pre-stimulus, windows sliding in $1 \mathrm{~ms}$ steps for a total of 1950 windows), and the distribution of post-stimulus spike rates of all Pyr neurons (in the $5-55 \mathrm{~ms}$ window post stimulus) across 200 randomized microcircuits. The intersection point between the two distributions was set as the stimulus detection threshold. The probability of false detection was calculated by the integral of the pre-stimulus distribution above the detection threshold divided by the integral of the entire pre-stimulus distribution. The probability of failed detection was calculated by the integral of the post-stimulus distribution below the detection threshold divided by the integral of the entire post-stimulus distribution.

Statistical Tests. We determined statistical significance using paired-sample or two-sample t-test, where appropriate. We calculated the effect size using Cohen's $d$ (the difference in means divided by the pooled standard deviation).

\section{Acknowledgements}

HKY, AGM, FM and EH thank the Krembil Foundation for funding support. HKY and EH were also supported by a stipend award from the Department of Physiology at University of Toronto.

\section{References}

1. Gentet, L. J. et al. Unique functional properties of somatostatin-expressing GABAergic neurons in mouse barrel cortex. Nature Neuroscience 15, 607-612 (2012).

2. Tremblay, R., Lee, S. \& Rudy, B. GABAergic Interneurons in the Neocortex: From Cellular Properties to Circuits. Neuron 91, 260-292 (2016).

3. Northoff, G. \& Sibille, E. Why are cortical GABA neurons relevant to internal focus in depression? A cross-level model linking cellular, biochemical and neural network findings. Molecular Psychiatry 19, 966-977 (2014).

4. Prévot, T. \& Sibille, E. Altered GABA-mediated information processing and cognitive dysfunctions in depression and other brain disorders. Molecular Psychiatry 1-17 (2020) doi:10.1038/s41380-0200727-3.

5. Lewis, D. A., Hashimoto, T. \& Volk, D. W. Cortical inhibitory neurons and schizophrenia. Nature Reviews Neuroscience 6, 312-324 (2005).

6. Levinson, A. J. et al. Evidence of Cortical Inhibitory Deficits in Major Depressive Disorder. Biological Psychiatry 67, 458-464 (2010).

7. Fuchs, T. et al. Disinhibition of somatostatin-positive GABAergic interneurons results in an anxiolytic and antidepressant-like brain state. Mol Psychiatry 22, 920-930 (2017). 
8. Duman, R. S., Sanacora, G. \& Krystal, J. H. Altered Connectivity in Depression: GABA and Glutamate Neurotransmitter Deficits and Reversal by Novel Treatments. Neuron 102, 75-90 (2019).

9. Lin, L.-C. \& Sibille, E. Reduced brain somatostatin in mood disorders: a common pathophysiological substrate and drug target? Front Pharmacol 4, 110 (2013).

10. Fee, C. et al. Behavioral deficits induced by somatostatin-positive GABA neuron silencing are rescued by alpha 5 GABA-A receptor potentiation. International Journal of Neuropsychopharmacology (2021) doi:10.1093/ijnp/pyab002.

11. Seney, M. L., Tripp, A., McCune, S., A. Lewis, D. \& Sibille, E. Laminar and cellular analyses of reduced somatostatin gene expression in the subgenual anterior cingulate cortex in major depression. Neurobiology of Disease 73, 213-219 (2015).

12. Prevot, T. D. et al. Novel Benzodiazepine-Like Ligands with Various Anxiolytic, Antidepressant, or ProCognitive Profiles. CXP 5, 84-97 (2019).

13. Ali, A. B. \& Thomson, A. M. Synaptic alpha 5 subunit-containing GABAA receptors mediate IPSPS elicited by dendrite-preferring cells in rat neocortex. Cereb Cortex 18, 1260-1271 (2008).

14. Obermayer, J. et al. Lateral inhibition by Martinotti interneurons is facilitated by cholinergic inputs in human and mouse neocortex. Nat Commun 9, (2018).

15. Silberberg, G. \& Markram, H. Disynaptic Inhibition between Neocortical Pyramidal Cells Mediated by Martinotti Cells. Neuron 53, 735-746 (2007).

16. Prevot, T. D. et al. Reversal of Age-Related Neuronal Atrophy by a5-GABAA Receptor Positive Allosteric Modulation. Cerebral Cortex (2020) doi:10.1093/cercor/bhaa310.

17. Hodge, R. D. et al. Conserved cell types with divergent features in human versus mouse cortex. Nature 573, 61-68 (2019).

18. Molnár, G. et al. Human pyramidal to interneuron synapses are mediated by multi-vesicular release and multiple docked vesicles. eLife 5, e18167 (2016).

19. Komlósi, G. et al. Fluoxetine (Prozac) and Serotonin Act on Excitatory Synaptic Transmission to Suppress Single Layer 2/3 Pyramidal Neuron-Triggered Cell Assemblies in the Human Prefrontal Cortex. J Neurosci 32, 16369-16378 (2012).

20. Seeman, S. C. et al. Sparse recurrent excitatory connectivity in the microcircuit of the adult mouse and human cortex. eLife 7, e37349 (2018).

21. Mohan, H. et al. Dendritic and Axonal Architecture of Individual Pyramidal Neurons across Layers of Adult Human Neocortex. Cereb Cortex 25, 4839-4853 (2015).

22. Gidon, A. et al. Dendritic action potentials and computation in human layer $2 / 3$ cortical neurons. Science 367, 83-87 (2020).

23. Beaulieu-Laroche, L. et al. Enhanced Dendritic Compartmentalization in Human Cortical Neurons. Cell 175, 643-651.e14 (2018).

24. Chameh, H. M. et al. Sag currents are a major contributor to human pyramidal cell intrinsic differences across cortical layers and between individuals. bioRxiv 748988 (2019) doi:10.1101/748988.

25. Gouwens, N. W. et al. Systematic generation of biophysically detailed models for diverse cortical neuron types. Nature Communications 9, 710 (2018).

26. Zurita, H., Feyen, P. L. C. \& Apicella, A. J. Layer 5 Callosal Parvalbumin-Expressing Neurons: A Distinct Functional Group of GABAergic Neurons. Front. Cell. Neurosci. 12, (2018).

27. Prönneke, A. et al. Characterizing VIP Neurons in the Barrel Cortex of VIPcre/tdTomato Mice Reveals Layer-Specific Differences. Cereb Cortex 25, 4854-4868 (2015).

28. Ma, Y., Hu, H., Berrebi, A. S., Mathers, P. H. \& Agmon, A. Distinct Subtypes of Somatostatin-Containing Neocortical Interneurons Revealed in Transgenic Mice. J. Neurosci. 26, 5069-5082 (2006).

29. Hay, E., Hill, S., Schürmann, F., Markram, H. \& Segev, I. Models of Neocortical Layer 5b Pyramidal Cells Capturing a Wide Range of Dendritic and Perisomatic Active Properties. PLOS Computational Biology 7, e1002107 (2011). 
30. Scimemi, A. et al. Tonic GABAA receptor-mediated currents in human brain. European Journal of Neuroscience 24, 1157-1160 (2006).

31. Florez, C. M. et al. In Vitro Recordings of Human Neocortical Oscillations. Cerebral Cortex 25, 578-597 (2015).

32. Grin-Yatsenko, V. A., Baas, I., Ponomarev, V. A. \& Kropotov, J. D. EEG Power Spectra at Early Stages of Depressive Disorders. Journal of Clinical Neurophysiology 26, 401-406 (2009).

33. Colombo, M. A. et al. The spectral exponent of the resting EEG indexes the presence of consciousness during unresponsiveness induced by propofol, xenon, and ketamine. Neurolmage 189, 631-644 (2019).

34. Halgren, M. et al. The generation and propagation of the human alpha rhythm. PNAS 116, 2377223782 (2019).

35. Huang, H., Thompson, W. \& Paulus, M. P. Computational Dysfunctions in Anxiety: Failure to Differentiate Signal From Noise. Biol Psychiatry 82, 440-446 (2017).

36. Koetsier, G. C. et al. CPT performance in major depressive disorder before and after treatment with imipramine or fluvoxamine. Journal of Psychiatric Research 36, 391-397 (2002).

37. McDermott, L. M. \& Ebmeier, K. P. A meta-analysis of depression severity and cognitive function. Journal of Affective Disorders 119, 1-8 (2009).

38. Blier, P. \& El Mansari, M. Serotonin and beyond: therapeutics for major depression. Philosophical Transactions of the Royal Society B: Biological Sciences 368, 20120536 (2013).

39. Philip, N. S., Carpenter, L. L., Tyrka, A. R. \& Price, L. H. Nicotinic acetylcholine receptors and depression: a review of the preclinical and clinical literature. Psychopharmacology 212, 1-12 (2010).

40. Lee, S., Hjerling-Leffler, J., Zagha, E., Fishell, G. \& Rudy, B. The Largest Group of Superficial Neocortical GABAergic Interneurons Expresses Ionotropic Serotonin Receptors. J. Neurosci. 30, 16796-16808 (2010).

41. Kang, H. J. et al. Decreased expression of synapse-related genes and loss of synapses in major depressive disorder. Nature Medicine 18, 1413-1417 (2012).

42. Koenigs, M. \& Grafman, J. The functional neuroanatomy of depression: Distinct roles for ventromedial and dorsolateral prefrontal cortex. Behav Brain Res 201, 239-243 (2009).

43. Keller, A. J. et al. A Disinhibitory Circuit for Contextual Modulation in Primary Visual Cortex. Neuron 108, 1181-1193.e8 (2020).

44. Yu, J., Hu, H., Agmon, A. \& Svoboda, K. Recruitment of GABAergic Interneurons in the Barrel Cortex during Active Tactile Behavior. Neuron 104, 412-427.e4 (2019).

45. Hay, E. \& Segev, I. Dendritic Excitability and Gain Control in Recurrent Cortical Microcircuits. Cereb Cortex 25, 3561-3571 (2015).

46. Peng, Y. et al. High-throughput microcircuit analysis of individual human brains through nextgeneration multineuron patch-clamp. elife 8, e48178 (2019).

47. Harris, J. A. et al. Hierarchical organization of cortical and thalamic connectivity. Nature 575, 195-202 (2019).

48. Laubach, M., Amarante, L. M., Swanson, K. \& White, S. R. What, If Anything, Is Rodent Prefrontal Cortex? eNeuro 5, (2018).

49. Hagen, E., Næss, S., Ness, T. V. \& Einevoll, G. T. Multimodal Modeling of Neural Network Activity: Computing LFP, ECoG, EEG, and MEG Signals With LFPy 2.0. Front. Neuroinform. 12, (2018).

50. Mäki-Marttunen, T. et al. Alterations in Schizophrenia-Associated Genes Can Lead to Increased Power in Delta Oscillations. Cereb Cortex 29, 875-891 (2019).

51. Hawrylycz, M. J. et al. An anatomically comprehensive atlas of the adult human brain transcriptome. Nature 489, 391-399 (2012).

52. 2010 Allen Institute for Brain Science. Allen Human Brain Atlas. Available from: human.brain-map.org. 
53. Asgari, A. et al. Low-frequency electrical stimulation enhances the effectiveness of phenobarbital on GABAergic currents in hippocampal slices of kindled rats. Neuroscience 330, 26-38 (2016).

54. Schulz, J. M., Knoflach, F., Hernandez, M.-C. \& Bischofberger, J. Dendrite-targeting interneurons control synaptic NMDA-receptor activation via nonlinear $\alpha 5-$ GABAA receptors. Nat Commun 9, 3576 (2018).

55. Carnevale, N. T. \& Hines, M. L. The NEURON Book. (Cambridge University Press, 2006). doi:10.1017/СВO9780511541612.

56. Hashemi, E., Ariza, J., Rogers, H., Noctor, S. C. \& Martínez-Cerdeño, V. The Number of ParvalbuminExpressing Interneurons Is Decreased in the Prefrontal Cortex in Autism. Cereb Cortex 27, 1931-1943 (2017).

57. Krienen, F. M. et al. Innovations present in the primate interneuron repertoire. Nature 586, 262-269 (2020).

58. Van Geit, W. et al. BluePyOpt: Leveraging Open Source Software and Cloud Infrastructure to Optimise Model Parameters in Neuroscience. Front. Neuroinform. 10, (2016).

59. Hay, E., Schürmann, F., Markram, H. \& Segev, I. Preserving axosomatic spiking features despite diverse dendritic morphology. J Neurophysiol 109, 2972-2981 (2013).

60. Larkman, A. U. Dendritic morphology of pyramidal neurones of the visual cortex of the rat: III. Spine distributions. J Comp Neurol 306, 332-343 (1991).

61. Holmes, W. R. \& Rall, W. Estimating the electrotonic structure of neurons with compartmental models. J Neurophysiol 68, 1438-1452 (1992).

62. Ponce, M. et al. Deploying a Top-100 Supercomputer for Large Parallel Workloads: the Niagara Supercomputer. in Proceedings of the Practice and Experience in Advanced Research Computing on Rise of the Machines (learning) 1-8 (Association for Computing Machinery, 2019). doi:10.1145/3332186.3332195.

63. Loken, C. et al. SciNet: Lessons Learned from Building a Power-efficient Top-20 System and Data Centre. J. Phys.: Conf. Ser. 256, 012026 (2010).

64. Sivagnanam, S. et al. Introducing the neuroscience gateway. CEUR Workshop Proceedings 993, (2013).

65. Ramaswamy, S. et al. The neocortical microcircuit collaboration portal: a resource for rat somatosensory cortex. Front Neural Circuits 9, (2015).

66. Fuhrmann, G., Segev, I., Markram, H. \& Tsodyks, M. Coding of temporal information by activitydependent synapses. J Neurophysio/ 87, 140-148 (2002).

67. Szegedi, V. et al. Plasticity in Single Axon Glutamatergic Connection to GABAergic Interneurons Regulates Complex Events in the Human Neocortex. PLoS Biol 14, (2016).

68. Markram, H. et al. Reconstruction and Simulation of Neocortical Microcircuitry. Cell 163, 456-492 (2015).

69. Bryson, A. et al. GABA-mediated tonic inhibition differentially modulates gain in functional subtypes of cortical interneurons. PNAS 117, 3192-3202 (2020).

70. Teleńczuk, B. et al. Local field potentials primarily reflect inhibitory neuron activity in human and monkey cortex. Sci Rep 7, 40211 (2017).

71. Destexhe, A., Rudolph, M., Fellous, J. M. \& Sejnowski, T. J. Fluctuating synaptic conductances recreate in vivo-like activity in neocortical neurons. Neuroscience 107, 13-24 (2001).

72. Welch, P. The use of fast Fourier transform for the estimation of power spectra: A method based on time averaging over short, modified periodograms. IEEE Transactions on Audio and Electroacoustics 15, 70-73 (1967).

73. Guet-McCreight, A. \& Skinner, F. K. Using computational models to predict in vivo synaptic inputs to interneuron specific 3 (IS3) cells of CA1 hippocampus that also allow their recruitment during rhythmic states. PLoS One 14, e0209429 (2019). 